\title{
$\widehat{A}$ Madridge \\ madridge Journal of Neuroscience \\ nterconnecting Scientific world
}

Research Article

Open Access

\section{Angry Faces may Capture Attention But do they Hold It?}

\author{
Laura Jenkins ${ }^{\star}$
}

Northumbria University, Department of Psychology, Newcastle-upon-Tyne, UK

\section{Article Info}

\author{
*Corresponding author:

\section{Laura Jenkins} \\ Northumbria University \\ Department of Psychology \\ Newcastle-upon-Tyne \\ UK \\ E-mail: laurajenkins840@gmail.com
}

Received: September 13, 2017

Accepted: September 27, 2017

Published: October 04, 2017

Citation: Jenkins L. Angry Faces may Capture Attention But do they Hold It? Madridge J Neurosci. 2017; 1(1): 7-16.

doi: $10.18689 / \mathrm{mjns}-1000103$

Copyright: @ 2017 The Author(s). This work is licensed under a Creative Commons Attribution 4.0 International License, which permits unrestricted use, distribution, and reproduction in any medium, provided the original work is properly cited.

Published by Madridge Publishers

\begin{abstract}
The Anger Superiority Effect refers to an individual's tendency to avoid an angry face after locating it in a crowd situation. Previous literature has used different methodologies, consisting of cartoon like facial images [9] and real life photographs [20] to look at this effect. The aim of the current study was to also look at the Anger Superiority Effect but in a different way to the past research. Researchers aim to use the method of eye tracking [20] to try and provide a new line of evidence towards Anger Superiority. 20 participants ( 7 male and 13 female) from the area of Newcastle-upon-Tyne were asked to complete a simple emotive face memory task whilst having their eye movements tracked. Researchers wanted to find out if participants avoided angry faces after locating them, and also if there were any areas of the angry faces themselves that were of particular interest. Results demonstrated that participants did not avoid the angry face after focusing on it, contradicting the previous literature. It was also demonstrated that angry faces were fixated less quickly than other emotions, again not supporting past literature. All results were discussed in relation to the past Anger Superiority studies and improvements in relation to future research were suggested.
\end{abstract}

Keywords: Angry face; Anger superiority effect; Eye tracking; Facial features.

\section{Introduction}

Anger Superiority refers to an individual's tendency to detect angry emotive faces more efficiently than any other emotive face, such as happy or sad ones. An angry face can be defined as one which expresses the emotion of anger. The face will have a ' $v$ ' like structure of the eyebrows and eyes, and the mouth will be closed. A happy face, however, contrasts this with a smile presented on the mouth and an arch shaped raised eyebrow. A neutral face is one which has no emotive expression.

Hansen and Hansen (1988) first reported Anger Superiority and focussed upon the situation of a crowd for their research [10]. Researchers conducted three small experiments of which they aimed to look at the proposed 'pop out' effect of threatening faces. In this case, the angry faces were seen as the threatening ones. For each experiment, participants were presented with a crowd like image consisting of nine faces. Participants were then asked if a discrepant face, an angry face, had been presented in the image (for example, an angry face amongst a happy crowd). For fifty percent of the trials, a discrepant face had been present whereas in the other fifty percent, there was no discrepant face. As there were three different experiments, one small aspect of each experiment had been manipulated. In experiment one, participants were shown arrays consisting of nine different faces. In experiment two, participants were shown arrays consisting of nine identical faces. In experiment three, the size of the crowd were changed. For this experiment, participants had to look at crowds of both four and nine faces so that the existence of the 'face in the crowd' effect could be tested with the 
presence of angry faces. Results from all three experiments gave support for both the 'pop-out' effect and the anger superiority effect. All three experiments demonstrated that participants were able to identify the angry discrepant face more easily than if the discrepant face was a happy or a neutral emotion. The size of the crowds (either four or nine faces) also demonstrated a difference. When being presented with a crowd consisting of only four faces, participants took less time to locate the discrepant face. This indicated that participants were using a serial search technique and when the discrepant face was found, participants would not go back to check the crowd again before answering yes or no to the researcher. The size of the crowd was only being used to show support for the anger superior effect, and although a difference in detection times were recorded, results for this difference were not discussed in detail. Researchers only wanted to see if any pop out effects were present and not any specific differences between the effects and detection times.

Similarly to Hansen and Hansen (1988), Fox, Lester, Russo, Bowles, Pichler and Dutton (2000) also investigated how angry faces can be detected more easily [10]. Researchers highlighted Hansen and Hansen (1988) as a very important piece of literature because it gave reasons to believe that face processing was automatic, and highlighted the importance of pre-attentive processing [10]. This is where people will automatically accumulate information from the environment (or other distracters on the computer screen) to help make decisions, in this case the detection of an angry face. Fox et al. (2000) also gave indications of inconsistent results in background research relating to Hansen and Hansen (1988) $[9,10]$. Researchers found a study conducted by Nothdurft (1993) who also looked at whether angry faces 'pop out' from other emotive faces [16]. Nothdurft (1993) looked at cartoonlike schematic faces and found that there was no bias for angry faces in the search tasks [16]. This does not replicate findings from Hansen and Hansen (1988) and questions surrounding the presentation of an anger superiority effect (and anger pop out) still remain [10].

These inconsistent results were all related to the search slopes of the faces and the reaction times of any visual search tasks (all created in different ways for each study), making it difficult to compare each investigation. Therefore, Fox et al. (2000) decided to create their own methodology to investigate the face in the crowd effect and conducted four smaller experiments to investigate this [9]. Reaction times were recorded in all four experiments and participants had to simply decide if all faces presented were the same or different (this would allow researchers to find if participants had found the angry face displayed.). Experiment one was designed to simply replicate the face in the crowd effect by using four faces placed in a circular pattern. Experiment two had the same aims as experiment one, however, the presentation times of the faces were $800 \mathrm{~ms}$ instead of $300 \mathrm{~ms}$. Experiment three looked at the different orientations of faces (for example, inverted faces) and experiment four focussed on the curvatures on the faces (the upward curve on a happy face and the downward curve on the angry or sad face). Results from the investigation provided evidence for the face in the crowd effect and demonstrated that participants detected the angry face quicker than any other emotive face.

Similar to Fox et al. (2000), Tipples, Atkinson and Young (2002) looked at angry faces and highlighted the importance of facial features such as an eyebrow frown $[9,27,28]$. Researchers conducted several mini experiments where participants had to view arrays of both faces and objects containing an eyebrow shape, and then had to decide if all faces or objects were the same. These arrays consisted of nine faces or objects which resembled a crowd situation. The shapes and faces contained ' $v$ ' shape eyebrows as in an angry face and then ' $\wedge$ ' shaped eyebrows as in surprised or happy faces. This experiment, unlike the past research, focussed on schematic faces (simple line drawings displaying emotions). These faces mean that outside influences such as hair and background images could not influence participants' results in any way, but can in theory lack ecological validity. The results form Tipples, Atkinson and Young (2002) were in line with previous literature and demonstrated that participants detected the angry faces, especially with the ' $v$ ' shaped eyebrows more efficiently than the other objects or faces [27, 28]. Researchers highlighted that this ' $v$ ' shaped eyebrow was a very important feature to look at when being concerned with the detection of angry faces and noted that it was difficult for participants to detect the angry face when eyebrows were removed. However, despite supporting theories such as the Anger Superiority effect, this study can be questioned about its ecological validity. In real life the majority of individuals would not be seen without eyebrows, therefore this study cannot reflect a real life situation and can only provide researchers with a brief overview of the important features in the human face. This can also be said for research conducted by Fox and Damjanovic (2006) who investigated the importance of eyes and the mouth upon a threat superiority effect. Results of the current study were then replicated by Aviezer et al. (2008) who supported ideas that the ' $v$ ' shape of an angry face is a very important feature in anger avoidance strategies [4]. Aviezer et al. (2008), however, used real life images of faces attached to congruent (or incongruent) bodies to note whether participants could still identify the angry face [4]. Results did show support for an anger bias, and researchers concluded that people will still read information from the face even when surrounding information (like body gestures) are present. The research discussed above highlighted the importance of two facial regions, the mouth and the eyes. After focussing upon the avoidance of angry faces, current researchers aim to answer a second research question by looking at the eye movements related to the mouth and eyes of the angry faces. Current researchers will question whether people spend more time looking at the eye or mouth region for the angry faces.

Horstmann and Bauland (2006) took note of the lack of ecological validity of previous studies, therefore conducted experiments using real life faces taken from Young, Perrett, Calder, Sprengelmeyer, and Ekman (2002) [11, 28]. Similar to previous studies, the current study carried out four smaller 
experiments which had slightly different aims. Experiment one looked at a happy and angry face as a whole. Experiment two was similar to experiment one, however the model was a woman instead of a man and the emotive expressions appeared more prominent to participants. Experiments $3 a$ and $3 \mathrm{~b}$ simply looked at a salient facial feature. This was the mouth. Finally, experiment $4 a$ and $4 b$ focussed upon the orientation of the faces and participants viewed inverted faces instead of upright faces. Participants were shown a variety of array sizes, consisting of 1 face, 6 faces or 12 faces of which they had to try and identify a target face and ideally ignore the distracter faces. This was similar to the previous studies discussed above. The results of Horstmann and Bauland (2006) were in line with Fox et al. (2000) and Tipples, Atkinson and Young (2002) who also noted that participants could detect the angry face more efficiently $[9,11,27,28]$. The current study, however, discussed results in relation to the threat detector hypothesis [2] and not the Anger Superiority Effect which states that people can detect a threatening face more efficiently that other stimuli with the idea of them avoiding the face to stay away from any danger. Tipples, Atkinson and Young (2002) had also highlighted the eye area as an important feature in the face, however, there has been limited research focussing on both the eye and mouth areas, therefore a discrepancy still remains about the importance of each area of the face [28].

Ackerman, Shapiro, Neuuberg, Kenrick, Becker, Griskevicius, Maner and Schaller (2006) gave evidence towards anger avoidance theories but also proposed the theory that anger avoidance has an out-group heterogeneity bias [1]. This means that people may identify angry faces more efficiently if they are presented as a 'rival' group or even if the images were being presented as a different ethnic group. Researchers used a methodology that was slightly different to the previous literature detailed above. Participants had to watch a slideshow presentation which contained emotive faces and distracter faces, and then after watching a five minute distracter film clip, participants had to decide if they had seen (or not seen) individual faces that were then presented in succession to each other. Results from this literature demonstrated that once again, participants were able to identify the angry face quicker than a non-angry face. The literature aimed to look at different ethnic groups and highlighted an out group bias where white participants were able to identify black angry faces the quickest. This study can only be used for support for the anger superiority effect and cannot be used to shape the methodology of the current investigation. The reason behind this is that Ackerman et al [1]. (2006) had a completely different methodology to the previous studies; therefore the results of the current study cannot be compared based on methodological issues. Despite this, Ackerman et al [1]. (2006) still gave support for the anger superiority effect and were able to highlight the importance of ethnic groups in their research.

Both Mather and Knight (2006) and Ashwin, Wheelwright and Baron-Cohen (2006) used different samples to investigate the anger superiority effect $[3,15]$. Mather and Knight (2006) used a series of nine schematic faces to look at anger superiority in older adults [15]. As with Fox et al. (2000) and Tipples, Atkinson and Young (2002), Mather and Knight (2006) used grids of nine faces involving one discrepant face $[9,15$, $27,28]$. Participants were asked if all nine faces looked the same, and similar to previous studies [11], reaction times were recorded. Mather and Knight (2006) gave support for anger superiority and demonstrated that this effect does not decline with age as previous literature may have suggested [18]. Ashwin, Wheelwright and Baron-Cohen (2006) on the other hand, looked at anger superiority in Asperger Syndrome [3, $11,15]$. Researchers used a face in the crowd methodology and demonstrated that the individuals, who had been diagnosed with Asperger Syndrome, could also detect angry faces more efficiently, even if it was not as quick as the control group. Ashwin, Wheelwright and Baron-Cohen (2006) conducted four smaller experiments of which the types of distracters were manipulated during each experiment (ranging from all neutral distracters to no neutral distracters) [3]. Researchers concluded that the slightly slower reaction times of the individuals with Asperger Syndrome could suggest different face processing styles of people with this syndrome. However, the current research will not be focussing on individuals with Asperger Syndrome, therefore Ashwin, Wheelwright and Baron-Cohen (2006) can simply be quoted for supplying more evidence towards the quicker detection of angry faces (using the face in the crowd effect) [3].

Lipp, Price and Tellegen (2009) looked at the detection of angry, sad and happy faces and found a bias for the detection of the angry faces [12]. Participants took part in three smaller experiments which used both schematic faces and real life photographs. Participants had to view matrices consisting of nine faces and had to decide if all nine faces were the same, as in previous literature. In these matrices, there was one face which was different to the others in half of the trials. The trials consisted of happy, sad, neutral and angry faces and each 'different' face was presented in matrices of the other three emotive faces, for example, an angry face would be presented with nine happy faces, nine neutral faces and then nine sad faces. Participants were found to respond quicker if the 'different' face was an angry one, therefore giving support for the anger superiority effect and anger bias theories.

Pinkham, Griffin, Baron, Sasson and Gur (2010) is one of the most recent studies to investigate anger superiority using a face in the crowd method. Twenty six undergraduate students took part in the study and were presented with a series of arrays consisting of nine faces. Out of the nine faces, there was again one discrepant face presented, for example, an angry face amongst a neutral crowd of faces. Pinkham et al. (2010) has reviewed the past literature (mostly discussed above) and had created their methodology based upon the fact that the past literature had lacked ecological validity with using schematic faces [20]. Researchers, therefore, decided to use real life faces, all with hair and ears still presented with the face. For the purposes of the investigation, participants were informed that this was a simple memory task and had to decide if all nine faces displayed (in each array) were the same or different. Reaction times were recorded. Pinkham et al. 
(2010) was the first study of its kind to use a visual search task that had high ecological validity [20]. It was also the first to use faces (and distracter faces) that were not identical to each other, therefore increasing the validity of the task. As a direction for future research, Pinkham et al. (2010) suggested that an eye tracking method could be used to help supply support for the anger superiority effect and the possible avoidance of angry faces [20]. The current researchers therefore decided to investigate the anger superiority effect using the method of eye tracking. This is because eye tracking has never been used in this situation before.

The research conducted by Pinkham et al. (2010) was the last study of this nature to focus specifically on anger superiority and the avoidance of angry faces using the face in the crowd technique in adults [20]. Since then research has focussed upon an anger bias [24] but did not quote the relevant anger superiority literature, therefore the current study will not focus upon this piece of research.

One further study, which followed the research conducted by Pinkham et al. (2010), was Rosset, Santos, Fonseca, Rondan, Poinso and Deruelle (2011) [20, 23]. Researchers quoted Pinkham et al. (2010) in their work and used a similar methodology consisting of arrays of faces [20]. The main difference of the research was that Rosset et al. (2011) had used children instead of adults, therefore had a clear rationale for using cartoon like faces instead of real life adult faces in their research [23]. Results did give support for the anger superiority effect; however, because previous research had lacked data with children, the present research [23] could not be fully compared to previous research like Fox et al. (2000) [9]. Despite this problem, the study still gave evidence that even in a clinical population, anger avoidance and anger superiority effects can occur from a very young age.

The question still remains, however, as to why past researchers have focussed upon angry faces in their experiments and not on other emotions such as surprise. One main reason looks at the fact that individuals have a bias towards the detection of angry faces because these faces can be seen as a threat in different aversive situations [17]. Öhman and Mineka proposed the idea of preparedness in relation to negative stimuli, such as angry and fearful faces. When an individual detect this negative stimuli, they can then prepare themselves to be removed from the situation and avoid the aversive stimuli. In the case of the current investigation, participants would theoretically avoid the angry faces within the crowd situation. Both Fox et al (2000) and Tipples, Atkinson and Young (2002) have used the work from Öhman as an explanation of why people have detected the angry face more efficiently in a crowd situation, therefore the current study will do the same $[9,17,27]$.

Öhman and Soares (1993) suggested that humans are hardwired for threat; therefore have involuntary controls over the fact that they can then avoid the threatening stimuli. Unconscious mechanisms in the brain allow individuals to rapidly detect threatening stimuli, therefore meaning that in an ideal situation, the individual would not become involved with the threatening situation. For the current study, in the case of angry faces, participants would have no control over avoiding the angry face.

Research conducted by Ekman (1992) highlighted the importance of the five basic emotions that individuals could detect [7]. These five basic were happiness, sadness, anger, fear, disgust and surprise. Ekman's work highlighted the emotion of anger as being an emotion that was most popular and a one that could be studied in detail [7]. The past literature, as detailed above, uses Ekman as a reference when discussing relations to angry faces, therefore the current study will use Ekman's work and images to investigate the angry faces [7].

After reviewing the past literature, current researchers have created a clear rationale for their study.

The aim of this investigation is also to look at the anger superiority effect and to look at the eye movements in relation to the angry faces themselves. An eye tracking method is an appropriate method to use as this can give definitive regions as to where a participant is looking. Eye tracking has successfully been used within working memory investigations [26] and also to look at the perception of negative and positive faces [6], however there is a lack of study upon specifically angry faces.

Current researchers aim to use the future direction suggested by Pinkham et al. (2010) who gave the idea of the use of eye tracking as a different method of looking at the anger superiority effect [20]. Eye tracking is a method that has never been used in the area of anger superiority; therefore researchers decided that this was an opening for further investigations. After reviewing past literature, the current researchers aim to create a methodology (a programme consisting of facial images) that is similar to those of the past literature, in particular, Fox et al. (2000) and Pinkham et al. $(2010)[9,20]$. Fox et al. (2000) used arrays consisting of faces displayed in a circular pattern and Pinkham et al. (2010) used arrays displayed in a square shape, therefore the current study will also use these types of arrays [9, 20]. Pinkham et al. (2010) was one of the most recent pieces of research to highlight the importance of using real life faces in this type of work to increase ecological validity therefore the current researchers will use real life faces and not cartoon-like schematic faces that have previously been discussed [20]. Researchers have chosen to use an eye tracking method so that each individual's eye movements can be looked at and analysed in relation to the arrays being displayed on the screen. This is a simple method that can highlight whether an anger superiority effect is present. Researchers have created two research questions for the purposes of this investigation. 1) Angry faces capture attention but do they hold it?, and 2) For the angry faces, do people spend more time looking at the eye region or the mouth region?

To help answer these research questions, four hypotheses have been proposed and results will be analysed in relation to these hypotheses. They are as follows:

1. Participants will tend to fixate on the angry face first (i.e. anger pop-out). 
2. After fixating on the angry face, participants will tend to direct attention to the non-angry faces, and spend more time on the opposite side of the array as the angry face.

3. Participants will spend more time looking at the eye region compared to the mouth region on the angry faces.

4. On the memory task, participants will have better memory performance for the angry face compared to other emotions.

\section{Method \\ Design}

A repeated measures design was used and there was only one condition. This condition contained four faces (one happy, one angry and six 'other' emotive faces). The independent variable therefore was the emotion type and this had three levels consisting of happy, angry and other. The dependent variables were: 1 ) The first saccade from each participant (eye movement from the central fixation cross to a face), 2) The section of the screen that participants focussed on (e.g. face 1 , 2, 3 or 4) and 3) The scores on the memory task itself.

\section{Participants}

20 participants were used for the purposes of this investigation. There were 7 males and 13 females recruited from the area of Newcastle-upon-Tyne. Participants had normal (or corrected normal) vision and because the testing phase involved eye tracking, participants must not have been sensitive to infra-red light.

\section{Materials}

An Eyelink 1000 eye tracker was used to track the eye movements of participants. This equipment was used to record the fixation times and dwell times of participants eye movements in relation to the emotive faces presented. The eye tracking equipment allowed the researcher to calibrate and then validate participant's eye movements, to the precise millimetre. Please see www.sr-support.com for the technical details of the eye tracking equipment. To create the face programme, the SR Research Experiment Builder was used and triggered so that eye movements could be tracked on the appropriate experimental trials.

Images taken from Young, Perrett, Calder, Sprengelmeyer, and Ekman (2002) and The Nottingham Face Database were used to help create the arrays of the four faces in each trial [28]. These images were displayed in a square pattern, similar to those of Fox et al. (2000) and Pinkham et al. $(2010)[9,20]$. In each trial there were four emotive faces (angry, happy, and two neutral faces) with a happy face being presented in the opposite corner to every angry face.

\section{Procedure}

Participants were given the participant information sheet to read and if they were still happy to take part in testing, a consent form was then signed. Participants were placed into the eye-tracking machine (resting their chin on the chin rest) and the participants' eye position was calibrated. The participant was asked if they were comfortable enough to carry on with testing. If the participant said yes then the testing phase began. The participant was asked to look at 3 practice trials before a series of 60 trial arrays (images consisting of four facial expressions on one screen at a time). Before each trial appeared, a fixation cross was displayed for $2000 \mathrm{~ms}$ so that participants' eye movements could be directed back to the centre of the screen. Each trial (encoding image) was shown for a total of $5000 \mathrm{~ms}$ seconds. After each image was presented, a single face (response image) was then presented and participants were asked 'Was this face displayed in this position in the image/trial just shown?'. Participants had to press 'the green button'on the computer keyboard if they wanted to answer yes to the question and 'the red button' on the computer keyboard to answer no to the question. At the end of the testing phase, participants were fully debriefed, told the real aims of the investigation and were reminded of their right to withdraw up until the point of analysing the results. Please see Figure 1 for an example of one experimental trial.

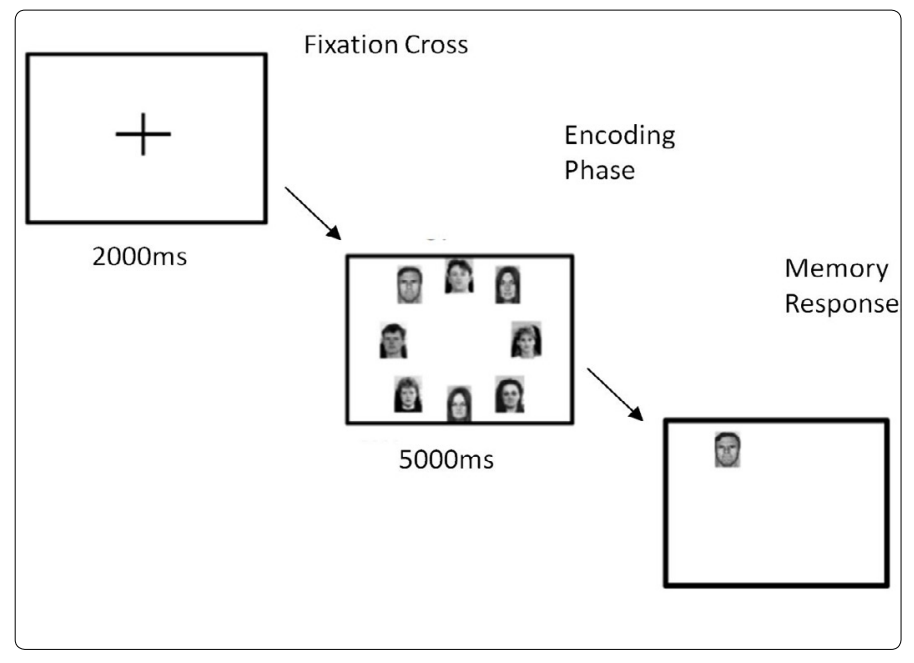

Figure 1. Example of one trial on the face memory task

\section{Results}

Eye tracking data viewer software was used to look at the data before it was transferred into a readable Microsoft Excel file for the creation of pivot tables.

Five one way repeated measures ANOVA's were then used to analyse the data for the Mean Total Dwell Times; the Mean First Fixation Times; the Score on the Face Memory Task; Reaction Times for Each Emotion, and Mouth and Eye Region Comparisons.

\section{Mean total dwell time}

Dwell time refers to the amount of time a participant fixates upon a certain point during the task. In this case, it refers to the amount of time that was spent looking at a certain emotive face. 
The total dwell times showed an effect of emotion $F(2,38)$ $=122.166, p<.001$. Participants spent more time looking at the angry faces ( $763 \mathrm{~ms} v 499 \mathrm{~ms}$ ) compared to the other faces, $t(19)=14.917, p<.001$. Participants also spent more time looking at the angry faces (763ms $\vee 723 \mathrm{~ms}$ ) compared to the happy faces alone, $t(19)=2.261, p<.001$ (see Table 1, below, for graphical representation).

This did not give support for hypothesis 2 and instead suggested that participants did not direct attention away from the angry face after locating it. Participants therefore, spent more time looking at the angry face during the experiment.

Table 1. Means and standard deviations (in milliseconds) of the total dwell time for each facial region.

\begin{tabular}{ccc}
\hline Angry Face & Happy Face & Other Face \\
\hline $746.51(192.25)$ & $706.69(197.93)$ & $488.60(125.89)$ \\
\hline
\end{tabular}

\section{Mean first fixation time}

Mean first fixation time refers to a participant's first eye movement and where they focus on first. In this case, mean first fixation time refers to which emotion the participant looked at first.

Maulchy's Test of Sphericity was shown to be not significant; therefore Huynh Feldt corrected results were reported.

The mean first fixation time showed a marginal effect of emotion $F(1.15,21.8)=5.99, p .019$. The angry faces were fixated less quickly than the other faces (1197 vs 1445), $t(19)=$ $-3.142, p=.005$. No other paired comparisons were significant (see Table 2, below, for graphical representation).

This did not give support for hypothesis 1 as participants did not fixate more quickly on the angry face. Instead, participants fixated on the other emotions more quickly.

Table 2. Means and standard deviations (in milliseconds) of the fixation time for each facial region.

\begin{tabular}{ccc}
\hline Angry Face & Happy Face & Other Face \\
\hline $3197.93(343.60)$ & $3381.26(312.97)$ & $3445.11(221.17)$ \\
\hline
\end{tabular}

\section{Score on face memory task}

Scores on the memory task were calculated by the eye tracking software to note any differences in recall of each emotion.

The scores on the face memory task showed a main effect of emotion on the task score. $F(5,95)=5.485, p<.001$. The significant differences were between the happy and sad emotions (.50 vs $.78, p=.006)$; the angry and sad emotions (.57 vs $.78, p=.014)$ and happy and disgusted emotions $(.50$ vs $.68, p=.011$ ). Results demonstrate that participants scored worse on the angry emotions compared to the sad emotions (all other pairs with anger were not significant). This means that researchers have not shown support for hypothesis 4 and instead have demonstrated that participants did not score higher for the angry faces.

\section{Reaction times for each emotion}

The reaction times for the happy, angry and other emotions were recorded using the eye tracking software before being transferred to a readable Microsoft Excel file.

Maulchy's Test of Sphericity was shown to be not significant; therefore, Huynh Feldt corrected results were reported for this ANOVA.

It was shown that emotion had no significant effect upon the reaction times, indicating that participants did not recognise the angry face any quicker than the other emotions, $F(4.85,92.17)=1.720, p=.140$. This does not show support for any of the four hypotheses as the angry faces did not have the quickest reaction times.

\section{Mouth and eye region comparison}

A comparison between the fixations on the mouth and eyes on the angry faces was made. A paired samples t-test was carried out on the data for the mouth and eye regions of the angry faces.

Results showed no significant difference between mean first fixation times of the mouth and eye regions of the angry face, $t(19)=-1.893, p=.074$ and $t(19)=-2.888, p=.777$.

This does not support hypothesis 3 as participants did not spend any more time looking at the eye region in comparison to the mouth region of the angry faces.

\section{Discussion}

The aim of the current research was to investigate the Anger Superiority Effect. This effect refers to an individual's tendency to detect angry emotive faces more efficiently than any other emotive face such as happy or sad ones. Researchers created a simple face memory programme and used an eye tracker to investigate participants' eye movements during the testing phases of the experiment. The method of eye tracking had never been used in the field of Anger Superiority, therefore researchers wanted to discover if this method could add any useful data to this psychological field.

Researchers created two research questions. The first 'angry faces capture attention but do they hold it?' wanted to discover if, as previous literature suggested [20], participants would avoid an angry face after locating it in a crowd situation. The second research question 'for the angry faces, do people spend more time looking at the eye region or mouth region?' wanted to give researchers more details regarding the angry faces being presented. Researchers wanted to discover whether the mouth region or eye region on the angry emotive faces were seen as the most important (and most looked at) aspect of the face.

Results demonstrated that participants spent more time looking at the angry faces compared to the other emotions, indicating that anger avoidance did not take place. It was also demonstrated that participants fixated on the angry face less quickly than the other emotions. This indicates that there was no evidence of an anger superiority effect. After focussing on 
the details of the angry faces, it can be said that both the eye and mouth regions have equal importance when looking at an angry face as there were no significant findings here. Results from the memory task indicated that participants did not find the angry faces any quicker than the other emotions and this was demonstrated by the non-significant reaction times of all emotive faces.

The first research question which was addressed was 'angry faces capture attention but do they hold it?' Researchers found an answer to this question although this was not in line with previous literature. It was concluded that the angry faces held attention instead of avoidance as the literature suggested. The current investigation was based on two studies who proposed ideas regarding anger superiority. Pinkham et al. (2010) had suggested the ideas of anger superiority and demonstrated this effect using grids of nine faces to represent a crowd situation. Fox et al. (2000) used circular patterns consisting of four to eight schematic drawn faces to show an anger superiority effect $[9,20]$. Current researchers used similar methodologies to the previous two studies but ultimately found differing results. It can be suggested that the slight difference in methodologies between the current study, Pinkham et al. (2010) and Fox et al. (2000) could be a reason why the current study did not show any evidence towards an anger superiority effect $[9,20]$. The study had only used grids of four faces, therefore this may not have been a true crowd situation [20]. If current researchers had used more than four faces then results may have differed and may have been more similar to the past literature. Because the current study only used four faces, participants had a lesser amount of faces to look at. Participants could have been looking simply at the amount of faces and the position of them, and not the emotions on the faces as we proposed.

The current research is also not in line with past research such as Tipples, Atkinson and Young (2002) and Ashwin, Wheelwright and Baron-Cohen (2006) [3, 27]. These studies had used the cartoon like drawings instead of the real life faces that has previously been used. The cartoon like faces are less ecologically valid but do contain less distracter features, like hair and ears, therefore could potentially be easier to search through in a memory task. It could be suggested that in the current study, participants paid more attention to the gender of participants, or the other features such as hair. Mather and Carstensen (2003) suggested that factors like age and gender of both participant and face shown could influence results [14]. If this was the case in the current study, then participants would not be focussing upon the emotion and would just be trying to remember the features of the faces for the memory task. Maybe a questionnaire at the end of the investigation would have given more information about participants' search techniques and memory techniques.

Results from the face memory task were not in line with the results from the eye tracking aspects of the current investigation. During the face memory task, participants did not score the highest for the angry faces, therefore indicating that there was no anger superiority present. If the angry faces had scored the highest then researchers could have suggested that this was due to the anger superiority effect, where participants were quick to locate the angry face before avoiding it. However, for the current study, this was not the case.

When looking at the results of the face memory task, there could be several reasons as to why researchers did not obtain the expected results. These reasons are concerned with the capacity of working memory and also the types of influences that can cause differences in memory.

Luck and Vogel (1997) looked at the capacity of working memory and suggested that human working memory can simply only store 3-4 items at one time [13]. This means that the current study did provide an adequate measure of memory as participants only had to memorise four faces at one time, however, results were simply just not as expected. Luck and Vogel (1997) had investigated this using squares, and not more detailed images like the faces used in the current study [13]. Research studies, conducted after this, began looking at face memory $[5,25]$. Scolari, Vogel and Awh (2008) proposed the idea that faces are more complex to memorise than simple objects [25]. This means that the current face memory task may have been seen as quite difficult compared to other memory tasks in the literature discussed above. Questions still remain about how complex faces really are in terms of memory. Do people focus upon the face as a whole and see this as one object, or do they simply pay attention to each individual feature? Further research using eye tracking techniques could be proposed to help address this issue. One way in which the current eye tracking research could be changed is in the way the memory task is presented to the participant. In previous literature [9] asked participants to look at a crowd of faces. Participants were then simply asked if all faces looked the same and did not have to look at a single emotive face presented after each array. If the current eye tracking study had created a face memory task that was similar to this, then results may have been more similar to the background literature discussed earlier.

Results from the current study can also not use Öhman and Mineka (2001) [18], Öhman and Dimberg (1978) or Öhman and Soares (1993) to explain any results. Researchers, here, gave explanations such as removing one's self from a threatening situation as a reason for wanting to avoid an angry face $[17,19,21,22]$. Current researchers demonstrated findings that contrasted these views and found that participants did not avoid the angry faces as it may have been suggested in the past literature. Previous discussions suggest that results could have been due to the differences in stimuli or even just the memory of the participants themselves.

The second research question was 'for the angry faces, do people spend more time looking at the eye region or mouth region?' This was also answered by the results of this investigation.

As previously mentioned, there was no support for any literature suggesting that the eye and mouth regions have different importance in angry facial expressions. Researchers 
believed that an eye tracking study would provide a new type of data towards this research question; however this was not the case. Issues with the different methodologies and images could have also caused the non-significant results in this case.

Fox et al. (2006) used a Photoshop computer programmes to manipulate images of emotive faces. Researchers, here, highlighted the eyes as important feature on the human face and therefore investigated this using pictures of eyes and then pictures of faces without eyes. Fox et al. (2006) concluded that the eyes provide a key feature to show threat and that the eyes ultimately make a person avoid the threatening face [9]. In the case of the current study, results did not support the findings of Fox et al. (2000) and did not highlight the eyes as an important feature [9]. From comparisons with the eye region and mouth region of the angry faces shown in the current study, it can be concluded that further investigation still need to be done to answer this research question.

Horstmann and Bauland (2006) also investigated important features in the face, and highlighted the mouth as an important feature when detecting faces with angry emotions. Here, researchers used cartoon like drawn faces to investigate the anger superiority effect, but instead of using oval shaped faces $[9,11]$, Horstmann and Bauland (2006) used circular shaped faces which had hair drawn on the faces. Results from the investigation provided evidence towards suggesting that the mouth was an important feature in detecting angry faces [11]. In experiment three of the investigation, researchers noted how an advantage of the mouth was seen but no advantage was seen for the eyes. Compared to the current eye tracking investigation, results do not show similarities. The current eye tracking study showed no advantage of either the mouth or eyes in relation to the angry faces that were presented. One question to note here would be concerning the asymmetries of the faces used by Horstmann and Bauland (2006) [11]. Because in experiment 3, the images were simply cartoon faces, they were drawn to be symmetrical. In a real life photograph, as used in the current study, facial features would rarely been seen as symmetric. It could be proposed, therefore, that the asymmetries in the faces (presented in the eye tracking investigation) were a way of distracting the participants from the aim of the study. Instead of looking at the emotions presented in the faces, participants could have been looking at the facial features themselves and could have been looking at any asymmetries present. If the current study was conducted again then a comparison of real life faces and cartoon like schematic faces could be made. Lipp, Price and Tellegen (2009) found that the detection of negative emotions (like anger) were more apparent when participants were being presented with the schematic faces. Although the real life photographs still provided evidence for the quicker detection of negative emotions, the effect was not as large as with the schematic faces compared to the real life faces. It can be questioned about whether an eye tracking study, using the same stimuli as Lipp, Price and Tellegen (2009), would produce similar results [12].
One way to investigate the research question proposed above would be to look at inverted emotive faces. This could be done simply as it has been done in the current study, with the only difference being the inversion of the faces. Researchers would be able to discover if participants were looking at the face as a whole of if they were looking at specific facial features, like the mouth or eyes. By inverting the faces, researchers may be forcing participants to look at the specific features of the face. If the faces were kept upright, participants would have the background knowledge of what a face looks like, and they may not pay attention to the smaller details of the face in order to memorise it. If participants were simply just using the mouth or eyes (of a given face) to decide if it was angry or happy, then surely inverting the face would not make any difference. Overall, participants would hopefully still be able to locate the angry face in a crowd situation no matter of any inversion.

Previous literature has also never used eye tracking in this situation before. This does not mean that eye tracking can no longer be used. It could simply mean that you could use eye tracking alongside the methods created by Fox et al. (2000) or Pinkham et al. (2010) $[9,20]$. These programmes have been validated and tested whereas the programme in the current study was not.

There are several areas that could be used for future research here. Firstly, during the testing phases of the investigation, it became apparent that participants knew the 'identity' of some of the faces involved. Because the emotive facial images had been taken from Young, Perrett, Calder, Sprengelmeyer, and Ekman (2002), the participants who had studied psychology (or facial related subjects) had previously seen these images before [28]. This could have affected the results of the current study as the psychology related participants could have been seen to have some advantage over the non-psychology participants. To resolve this issue, current researchers have suggested creating a whole new set of emotive facial images of which participants would have had no access to prior to testing. This could eliminate any form of bias in the results and would also eliminate any form of unfair advantage. Curby and Gauthier (2007) had investigated memory associated with faces and concluded that expertise of a face (or object) can affect results [5]. Researchers here looked at memory involving faces and objects and discovered that when participants had had previous experience with faces, they were more likely to receive a correct score on the face memory task. This could be said for the current study investigating anger superiority. Participants could have ignored the emotions in each face and could have simply looked at the faces which they knew. This gives a very good opportunity for future research as researchers would then have an unbiased set of results and any experience of the faces would make no difference. If a colour set of photographs was created, then this could also increase the ecological validity of the study as participants would be viewing real life colour photographs instead of the black and white photographs like Ekman has previously used. 
If an investigation like this was conducted again, researchers may be encouraged to use different sized crowds. Both Pinkham et al. (2010) and Fox et al. (2000) had used crowd situations consisting of up to nine faces, and found results of an anger superiority $[8,9,20]$. Results from the current investigation did not find results to support the anger superiority effect, therefore the next step would seem obvious - add more people into the crowd to look at the effect. However, researchers may need to question how many faces may be too many. Past literature has shown nine faces to be a good estimate of a crowd and this could be replicated again if the current researchers carried out further investigations. Adding more faces to the situation may increase the participants' chance of just guessing or not looking at the emotions present. Further investigation would need to be done to answer any questions relating to this matter.

When reviewing the past literature, above, one problem was noted. Several pieces of research had used cartoon-like schematic faces [11] whereas others had used real life faces [20]. There is one obvious difference here and this is that these two types of faces have very little in common. The schematic faces were drawn for the purpose of investigating such phenomenons like the anger superiority effect, however, the real-life faces were not. The real-life faces were either taken from Young, Perrett, Calder, Sprengelmeyer, and Ekman (2002) or new sets of emotive faces were created from pictures of living people [28]. Difficulties come when researchers try to compare research that has used the two different types of facial images. Surely a set of hand drawn cartoon like faces does not compare to a real photograph of a person? A suggestion for future research may be to use eye tracking to see if there are any comparable differences between the two types of faces. Of course, this may steer away from looking at anger superiority but would indeed give researchers a clearer idea of which type of facial image is best to use.

When conducting a piece of research, such as the one above, it is important to look at how the research can be applied to real life. This piece of research looked at anger avoidance and results could be used in many situations here. Firstly, researchers could consider the influence of social anxiety in the crowd situations. If an individual is suffering from social anxiety, then they may feel very uncomfortable looking at angry faces. If this study was conducted again using participants suffering from social anxiety, then results could be used to help develop techniques to decrease the effects of the social anxiety. For example, the current research looked at the influence of the eye and mouth regions of the angry faces. Participants suffering from social anxiety may not look at these regions in case the social anxiety is increased. If this is the case, participants could be taught different ways of managing their social anxiety and could be given some form of therapy which would help them to look at the more angry faces in a crowd situation.

In contrast to this, the current research could also be used in the case of anger management techniques. Because this study involved looking at angry faces, it could have provoked some form of angry memories for participants, therefore would not help anyone who suffered from anger issues. The study highlighted the fact that people do tend to focus longer on angry faces, and this could also be the case of people with anger issues. Techniques could be developed to help people manage their anger and pictures, like the ones used in the current study, could be used to help individuals learn how to deal with other people and situations that could cause angry emotions.

To conclude, this piece of research has given some insightful data to add to that of the field of Anger Superiority. Researchers were able to successfully create a face memory task and then track participants' eye movements during the completion of the task. Several ideas have been given for future research and these ideas can allow future researchers to incorporate more ideas into the area of eye tracking and anger superiority. Although results were not what researchers had hoped, improvements have been suggested if this investigation was to be carried out again.

\section{References}

1. Ackerman JM, Shapiro JR, Neuberg SL, Kenrick DT, Vaughn-Becker D, Griskevicius V, Maner JK, and Schaller. They All Look the Same to Me (Unless They're Angry): From Out-Group Homogeneity to Out-Group Heterogeneity. Psychological Science. 2006; 17(10): 836-840. doi: 10.1111/j.1467-9280.2006.01790.x

2. Aronoff J, Barclay AM, Stevenson LA. The recognition of threatening facial stimuli. J Pers Soc Psychol. 1988; 54(4): 647-655.

3. Ashwin C, Wheelwright S, Baron-Cohen S. Finding A Face In The Crowd: Testing The Anger Superiority Effect in Asperger Syndrome. Brain and Cognition. 2006; 61: 78-95. doi: 10.1016/j.bandc.2005.12.008

4. Aviezer H, Hassin RR, Ryan J, Grady C, Susskind J, Anderson A, Moscovitch M, Bentin S. Angry, Disgusted, or Afraid?: Studies on the Malleability of Emotion Perception. Psychological Science. 2008; 19(7): 724-732. doi: 10.1111/j.1467-9280.2008.02148.x.

5. Curby KM, Gauthier I. A Visual Short-Term Memory Advantage for Faces. Psychon Bull Rev. 2007; 14(4): 620-628.

6. Duque $A$, Vázquez C. Double attention bias for positive and negative emotional faces in clinical depression: Evidence from an eye tracking study. J Behav Ther Exp Psychiatry. 2015; 46: 107-114. doi: 10.1016/j. jbtep.2014.09.005

7. Ekman, P. Facial Expressions of Emotions: New Findings, New Questions. Psychological Science. 1992; 3(1): 34-38.

8. Fox E, Damjanovic L. The Eyes Are Sufficient to Produce a Threat Superiority Effect. Emotion. 2006; 6(3): 534-539. doi: 10.1037/1528-3542.6.3.534

9. Fox E, Lester V, Russo R, Bowles RJ, Pichler A, Dutton K. Facial Expressions of Emotion: Are Angry Faces Detected More Efficiently. Cognition and Emotion. 2000; 14(1): 61-92. doi: 10.1080/026999300378996

10. Hansen $\mathrm{CH}$, Hansen RD. Finding the Face In The Crowd: An Anger Superiority Effect. J Pers Soc Psychol. 1988; 54(6): 917-924.

11. Horstmann G, Bauland A. Search Asymmetries with Real Faces: Testing the Anger-Superiority Effect. Emotion. 2006; 6(2): 193-207. doi: 10.1037/15283542.6.2.193

12. Lipp OV, Price SM, Tellegen CL. Emotional faces in neutral crowds: Detecting displays of anger, happiness, and sadness on schematic and photographic images of faces. Journal of Motivation and Emotion. 2009; 33: $249-260$.

13. Luck SJ, Vogel EK. The Capacity of Visual Working Memory For Features and Conjunctions. Nature. 1997; 390: 279-281. doi: 10.1038/36846 
14. Mather M, Carstensen LL. Aging and Attentional Biases for Emotional Faces. Psychological Science. 2003; 14(5): 409-415. doi: 10.1111/14679280.01455

15. Mather M, Knight MR. Angry Faces Get Notices Quickly: Threat Detection is not Impaired Among Older Adults. J Gerontol B Psychol Sci Soc Sci. 2006; 61B (1): 54-57.

16. Nothdurft HC. Faces and facial expressions do not pop out. Perception. 1993; 22: 1287-1298. doi: 10.1068/p221287

17. Öhman A, Dimberg U. Facial Expressions as Conditioned Stimuli for Electrodermal Responses: A Case of 'Preparedness'?. J Pers Soc Psychol. 1978; 36(11): 1251-1258.

18. Öhman A, Mineka S. Fears, Phobias, and Preparedness: Toward an Evolved Module of Fear and Fear Learning. Psychological Review. 2001; 108(3): 483-522.

19. Öhman A, Soares JJF. On The Automatic Nature of Phobic Fear: Conditioned Electrodermal Responses to Masked Fear-Relevant Stimuli.J Abnorm Psychol. 1993; 102(1): 121-132.

20. Pinkham AE, Griffin M, Baron R, Sasson NJ, Gur RC. The Face in The Crowd Effect: Anger Superiority When Using Real Faces and Mulitple Identities. Emotion. 2010; 10(1): 141-146. doi: 10.1037/a0017387

21. Publication Manual of the American Psychological Association ( $6^{\text {th }}$ ed.) Washington, DC: APA.
22. Psychological Image Collection at Sterling (PICS). Nottingham Face Database.

23. Rosset D, Santos A, Da Fonseca D, Rondan CC, Poinso F, Deruelle C. More Than Just Another Face In The Crowd: Evidence From An angry Superiority. Research In Autism Spectrum Disorders. 2011; 5(2): 949-956. doi: 10.1016/j. rasd.2010.11.005

24. Schmidt-Daffy M. Modeling Automatic threat Detection: Development of a Face-in-the-Crowd Task. Emotion. 2011; 11(1): 153-168. doi: 10.1037/ a0022018

25. Scolari M, Vogel EK, Awh E. Perceptual Expertise Enhances the Resolution but not the Number of Representations in Working Memory. Psychonomic Bulletin and Review. 2008; 15(1): 215-222.

26. Tanaka T, Sugimoto $M$, Tanida $Y$, Saito $S$. The influences of working memory representations on long-range regression in text reading: an eye tracking study. Frontiers in human Neuroscience. 2014; 8: 765. doi: 10.3389/fnhum.2014.00765

27. Tipples J, Atkinson AP, Young AW. The Eyebrow Frown: A Salient Social Signal. Emotion. 2002; 2(3): 288-296.

28. Young A, Perrett D, Calder A, Sprengelmeyer R, Ekman P. Facial Expressions of Emotion - Stimuli and Tests (FEEST; 2002). London: Thames Valley Test. 